\title{
Caracterización de los Servicios de Atención en Violencia Familiar del área metropolitana de Buenos Aires, Argentina 1
}

\author{
Analysis of Services Targeting Family Violence \\ Against Women in Greater Metropolitan \\ Buenos Aires, Argentina 1
}

Roxana Cecilia Ynoub 2 1 Investigación realizada
con el apoyo de la Red de
Investigación en Sistemas
y Servicios de Salud en
el Cono Sur (auspiciada por
la Fundación Oswaldo Cruz,
de Brasil, y el International
Development Research Center,
de Canadá).
2 Instituto de Investigaciones,
Facultad de Psicología,
Universidad de Buenos Aires.
Güemes 729, Acasusso (1640),
Prov. Buenos Aires, Argentina.
A bstract This study presents a diagnosis and evaluation of the availability of services related to family violence against women in Greater Metropolitan Buenos Aires, Argentina. The paper focuses on institutional devel opment, theoretical foci, intervention modalities, and production of information and research. Existing networks and interprofessional rel ationships are also described. Results point to several noteworthy theoretical perspectives. Some programs include gender as an issue, while others do not. The relationship (more informal than formal) between governmental and nongovernmental organizations is also a rel evant issue. The paper concludes by proposing these institutional experiences as a model for the design of new public health policies.

Key words Domestic Violence; Women's Health; Health Systems; Public Policies

Resumen Se presentan, en estetrabajo, un diagnóstico y evaluación dela disponibilidad de servicios existentes en la regi ón metropolitana de Buenos Aires para la atención en Violencia Familiar Contra la Mujer. De el los se describen las redes existentes y las articulaciones intersectoriales. Asi mi smo se evaluan su desarrollo institucional, los enfoques teóricos, las modalidades de intervención y la producción de información e investigaciones. Entrelos resultados, se destaca la presencia de distintas perspectivas teóricas - particularmente entre quienes incl uyen o no la temática de género -, a las que corresponden distintas modali dades de intervención; y las conexiones - más informales que formal es - entre distintos sectores y áreas oficiales y las organizaciones no gubernamentales. Final mente se proponen las experiencias institucional es desarrol ladas en esta tema como un model o de nuevas modalidades en el diseño de políticas públicas y de salud. Palabras clave Violencia Doméstica; Salud de la Mujer; Si stemas de Salud; Políticas Públicas 


\section{Presentación y marco del proyecto}

La violencia familiar, y en particular la dirigida hacia la mujer, ha permanecido como una cuestión relegada al ámbito privado hasta hace no mucho tiempo. Recién en los últimos 25 años se la ha reconocido como problema social, y, paulatinamente, la atención y la preocupación han aumentado hasta tranformarse en tema prioritario a nivel internacional. Dicho reconocimiento emergió a inicio de los años 1970 de la mano de los movimientos defensores de los derechos de la mujer, y en el marco de una serie de reivindicaciones cuestionadoras de la relación entre los géneros y del papel de la mujer como sujeto y actor social (Nieves Rico, 1992).

Las Naciones Unidas incorporan decididamente el tema desde la década dedicada a la mujer (1975-1985). Es en ese período cuando la violencia familiar y hacia la mujer comienza a transformarse en asunto público. Desde entonces la ONU auspicia diversas reuniones de grupos de expertos sobre violencia contra la mujer, sigue el tema a través de su Comisión sobre la Condición de la Mujer, el Consejo Económico y Social, la Oficina de Estadísticas de las Naciones Unidas y el Comité de Prevención y Control del Delitos (Heise, 1994). Aunque no sin resistencias, desde entonces, la cuestión de la violencia familiar comenzó a ser incluída en los planes y programas de los gobiernos nacionales casi conjuntamente con la creación de áreas gubernamentales dedicadas a los asuntos de mujer y género.

En la región de las Américas, uno de los logros más importantes fue la llamada «Convención de Belém do Pará»- Convención Interamericana Para la Prevención, Erradicación y Sanción a la Violencia en Contra de las Mujeres -, sancionada en 1994 por la OEA y suscripta por todos los gobiernos de la región.

En Argentina fue el movimiento de mujeres, que se relanzó con el advenimiento de la democracia en 1983, el que instaló el tema en el país, y fueron las organizaciones no gubernamentales (ONGs) quienes crearon los primeros servicios de atención para mujeres víctimas de violencia. En lo que respecta al sector oficial, aunque el tema presentó las resistencias que suelen acarrear la mayoría de las reivindicaciones de género (por cuestionar los modelos tradicionales de la familia, por los estereotipos imperantes en funcionarios, magistrados y profesionales, etc.) y los problemas sociales en general (en el marco del progresivo abandono de los mismos por parte del Estado), tuvo mejor acogida que otros, como los de salud reproductiva: planificación familiar, aborto, etc. en los que hasta los organismos de mujer no tomaron partido o se mostraron refractarios a su tratamiento.

En tal sentido pueden reconocerse algunos avances, como la ratificación de la convención regional mencionada (1996), y la sanción (1994) y reglamentación (1996) de la Ley 24417, de "Protección contra la Violencia Familiar". Esta legislación, entre otras cosas, introduce una reforma en los procedimientos penales (autoriza al juez, en caso de denuncias por lesiones, ordenar la expulsión del hogar del agresor), extiende el concepto de grupo familiar a las uniones de hecho y obliga a denunciar hechos de maltrato familiar (entre los que incluye, además del maltrato físico, el psicológico). A partir de su reglamentación, se estipula la creación de una red de Centros de Información y Asesoramiento en el área de la Ciudad de Buenos Aires. A pesar de que esta legislación sólo tiene alcance federal (las provincias deben adherir por ley provincial, si desean incorporarla), sentó un precedente importante en la materia, en particular porque, para su reglamentación, se creó una instancia interinstitucional de áreas de gobierno, y se dio, además, cierta participación a representantes de organismos no gubernamentales.

Por otra parte, cabe mencionar que, aunque existan instancias nacionales dedicadas a los asuntos de la mujer (Subsecretaría de la Mujer, durante el período 1983-1989, y Consejo Nacional de la Mujer, desde 1991 hasta la fecha), los programas y políticas estuvieron y están principalmente diseñados desde los poderes locales (municipales y provinciales), resultando de ello una variedad de efectores aún dentro del mismo sector oficial que, en la gran mayoría de los casos, no mantienen coordinación ni integración entre sí.

En el área de la salud, desde hace al gunos años, se asiste a la aparición de estos servicios en hospitales públicos o en centros de atención primaria, aunque ellos han surgido, la mayoría de las veces de manera espontánea, como resultado de la organización de los profesionales demandados, antes que por la existencia de planes derivados de los centros de coordinación sanitarios. Sólo posteriormente se fueron creando instancias coordinadoras, como la actual red dependiente de la Dirección de Salud Mental del Gobierno de la Ciudad de Buenos Aires (aunque también ellas están circunscriptas a las áreas jurisdiccionales correspondientes).

Esta situación institucional hace que se torne difícil la evaluación de la magnitud de la problemática, del tipo y de las características 
de la población demandante, de la eficacia y eficiencia de la atención, de la oferta de servicios, etc. Cada una de las instituciones tiene distintos enfoques, criterios de abordaje y modalidades de registro, haciéndose aún más díficil el encuentro entre ellos para la producción de información confiable.

En base a ese estado de situación, la presente investigación se propuso producir una evaluación de la disponibilidad institucional existente en región metropolitana de Buenos Aires (Capital Federal y Partidos del Gran Buenos Aires), área que concentra el $30 \%$ del total de la población nacional, considerando la articulación interinstitucional (redes) e intersectorial de los Servicios (particularmente entre las organizaciones de la comunidad y el Estado), la historia de su desarrollo, su capacidad para la producción sistemática de información, y las potencialidades y obstáculos que se presentan en su funcionamiento.

Aún cuando se trata de un análisis de Servicios de Atención en Violencia Familiar (en el que se incluyen a veces a niños/as y ancianos/as), el eje del presente estudio ha sido puesto en la violencia contra la mujer, y, en especial, en la llamada violencia conyugal.

\section{Materiales y métodos}

La evaluación diagnóstica de los servicios de atención en violencia familiar incluyó los siguientes capítulos temáticos:

1) Descripción y caracterización de los servicios en términos de recursos humanos, concepciones teórico-prácticas de abordaje e inserción y desarrollo institucionales.

2) Evaluación de la articulación y del desarrollo de las redes institucionales existentes.

3) Evaluación de los alcances y de las modalidades en la producción de información por parte de los servicios, examinando su potencial aprovechamiento epidemiológico.

La unidad analítica central de la investigación fueron los Servicios de Atención. La información sobre los mismos se obtuvo en base a: a) un relevamiento por encuesta postal, enviada al total de instituciones dedicadas al tema, registradas en listados oficiales; b) entrevistas semi-estructuradas con informantes calificados del ámbito académico, gubernamental y no gubernamental; y c) una evaluación de los instrumentos de registro utilizados por las instituciones encuestadas (seleccionándose aquellas con mayor trayectoria en la producción de información para analizar su experiencia y metodología).
La información recogida a través de la encuesta postal fue analizada cuantitativa y cualitativamente. El análisis cualitativo estuvo circunscripto al material obtenido de las preguntas abiertas, ítems del cuestionario referidos a: Objetivos fundacionales (de la institución y/o del servicio); Definiciones de violencia doméstica; Caracterización de la población asistida; Obstáculos que se presentan en la práctica asistencial y en el desarrollo delas actividades de la institución.

Esta información fue ingresada a base de datos textual - Sistema Micro-Isis -, obteniéndose listados para cada uno de los ítems temáticos (corpus discursivos correspondientes a cada pregunta estímulo), que se evaluaron conjuntamente con variables contextuales como el "tipo de institución" (principalmente considerando la comparación entre organismos no gubernamentales y gubernamentales) y “Iocalidad” (ubicación geográfica). El análisis cualitativo estuvo centrado en la identificación de tipologías para los distintos temas indagados. El análisis cuantitativo se limitó a algunas pocas dimensiones, y, debido al escaso número de instituciones respondentes, las cifras sólo refieren tendencias posibles.

Se mantuvieron además entrevistas con informantes calificados (13), seleccionados por áreas de intervención: 2 del área académica (ambos tienen además participación directa en organizaciones no gubernamentales), 4 informantes que actúan en área "mujer" municipal, 1 que actúa en área "mujer" provincial, 1 del área hospitalaria, 2 de organizaciones no gubernamentales, 2 de policía, y una informante del área jurídica. La información aportada por ellos fue utilizada para completar y profundizar la recibida a través de la encuesta.

Finalmente, se recopilaron los instrumentos de registro utilizados por las instituciones encuestadas (en caso que tuvieran alguno), analizándose las variables e indicadores utilizados, su operacionalización y la producción de información resultante de los mismos.

\section{Resultados}

Servicios de atención en violencia familiar en el ámbito de la Capital Federal y del Gran Buenos Aires

El bajo índice de respuesta de la encuesta postal - aún cuando se realizaron tres envíos y se contactó telefónicamente a las instituciones constituye en sí mismo un dato a tener en cuenta. Sobre un total de 84 servicios registra- 
dos para el área de Capital Federal y Gran Buenos Aires, se recibieron 28 encuestas; ello significa que se cubrió aproximadamente un 33\% de las mismas. Cabe destacar sin embargo que, desde el punto de vista de la calidad de los servicios encuestados - si se excluye el sector salud -, respondieron casi el $100 \%$ de aquellos que son considerados referentes en la materia (Tabla 1).

En la Tabla 2, se presentan los «tipos de servicios» existentes en el área de cobertura (información producida a partir de las encuestas, informantes clave y fuentes secundarias), según su inserción y dependencia institucional, y se detallan los programas desarrollados por ellas.
Tabla 1

Servicios respondentes de la Encuesta Postal, por tipo de institución a la que pertenecen.

\begin{tabular}{lc}
\hline Tipo de institución & Cap. Fed. y GBA \\
\hline Organismo no gubernamental & 9 \\
Organismo gubernamental & $17\left(^{*}\right)$ \\
(excluídos salas u hospitales públicos) & \\
Hospitales o salas & - \\
Institución académica(**) & 1 \\
Dependiente de una organización religiosa & 1 \\
Total & 28 \\
\hline
\end{tabular}

Los «servi cios» ofrecidos por las instituciones encuestadas, en caso de que dispusieran de centros de atención, podían incluir: atención/asesoramiento psicológico; atención/asesoramiento teléfonico; grupos de ayuda mutua o de autoayuda; asesoramiento jurídico; patrocinio jurídico; y hogares-refugio (Tabla 3). La única institución que disponía de todos estos servicios conjuntamente fue la actual Dirección de la Mujer del Gobierno de la Ciudad de Buenos Aires (ex-Consejo Municipal); en los restantes casos, las combinaciones fueron variadas. Lo menos frecuente fueron el patrocinio jurídico y los refugios, mientras que la combinatoria más usual incluía asistencia psicológica, grupos de ayuda mutua y asesoramiento legal. Un sólo caso (organización no gubernamental) disponía de grupos para hombres violentos. Por su parte, la única institución religiosa respondente trataba el tema de manera reciente, no disponía de dispositivos asistenciales específicos, sino que lo abordaba en el marco de otras actividades desarrolladas con la comunidad. Otro de los servicios declarados casi por la totalidad de los encuestados entre gubernamentales (ONG) y no gubernamentales - fue él de prevención. En la mayoría de los casos, estaba dirigido al ámbito educativo - alumnos y docentes del nivel primario y medio - y, en segundo lugar, a la comunidad, a las áreas de salud y dependencias oficiales.

(*) Incluye dos servicios dependientes de la policía.

(**) No contaban con servicios propios.

Tabla 2

Servicios de atención en Violencia Doméstica, según dependencia institucional. Area metropolitana de Buenos Aires.

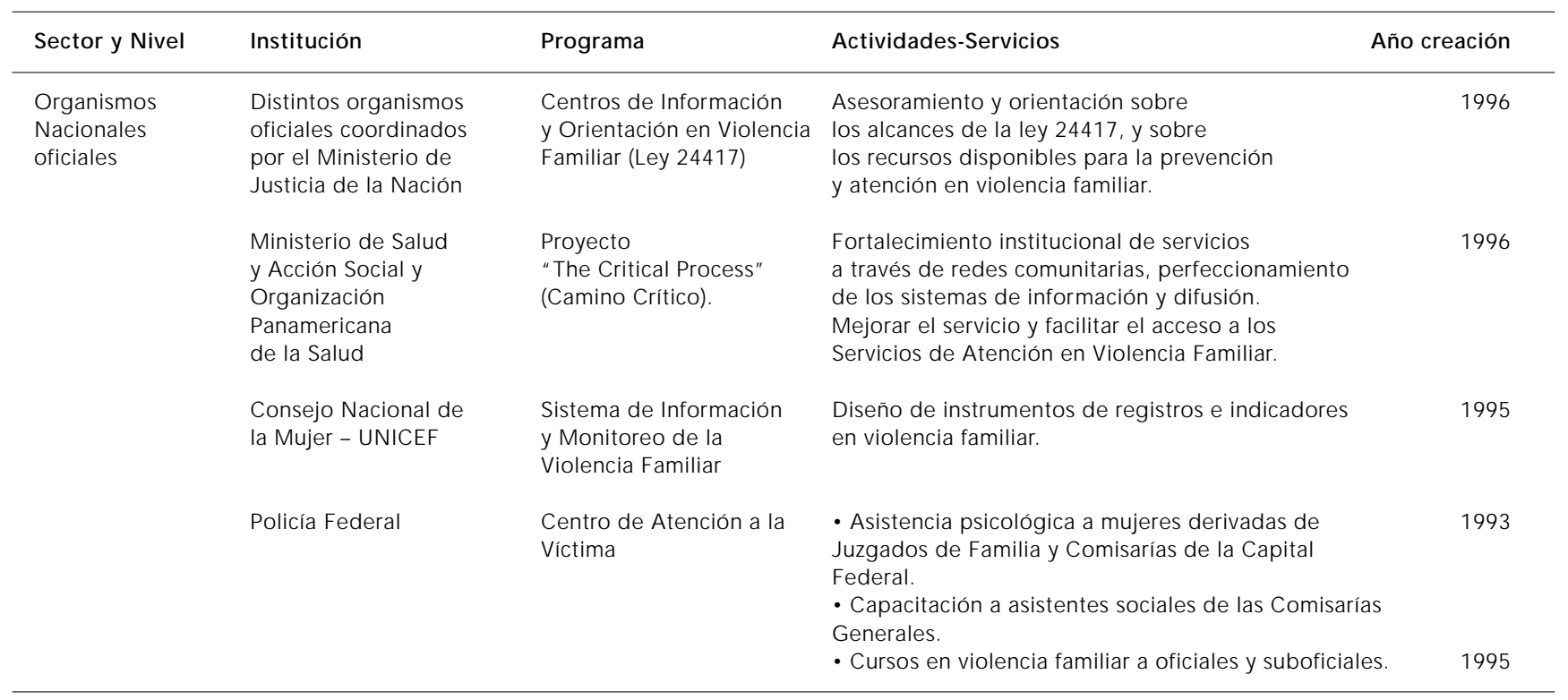


Tabla 2 (continuación)

Servicios de atención en Violencia Doméstica, según dependencia institucional. Area metropolitana de Buenos Aires.

\begin{tabular}{|c|c|c|c|c|}
\hline Sector y Nivel & Institución & Programa & Actividades-Servicios & ño creación \\
\hline \multirow[t]{2}{*}{$\begin{array}{l}\text { Organismos } \\
\text { provinciales ofi- } \\
\text { ciales (Provincia } \\
\text { de Buenos Aires) }\end{array}$} & $\begin{array}{l}\text { Consejo Provincial de } \\
\text { la Familia y Desarrollo } \\
\text { Humano }\end{array}$ & $\begin{array}{l}\text { - Programa de Prevención } \\
\text { de la Violencia Familiar } \\
\text { - Servicio de Atención } \\
\text { en Violencia Familiar }\end{array}$ & $\begin{array}{l}\text { - Asistencia y capacitación a organimos guberna- } \\
\text { mentales, no gubernamentales, educativos, etc, } \\
\text { en municipios. } \\
\text { - Programas de capacitación a policía. } \\
\text { - Asistencia psicológica, grupos de ayuda mutua, } \\
\text { asesoramiento jurídico. }\end{array}$ & 1989 \\
\hline & Policía Provincial & $\begin{array}{l}\text { - Comisarías de la Mujer. } \\
\text { (Actualmente } 6 \text { en funcio- } \\
\text { namiento) } \\
\text { - Programas de capaci- } \\
\text { tación de agentes. } \\
\text { (Convenio con el Consejo } \\
\text { Provincial de la Mujer) }\end{array}$ & $\begin{array}{l}\text { - Atención en violencia doméstica y/o sexual. } \\
\text { - Atención psicológica y asesoramiento legal. } \\
\text { En algunos casos, coordinan acciones con áreas } \\
\text { de Acción Social o UCEFF Municipales. } \\
\text { - Capacitación de agentes en Comisarías generales } \\
\text { para la atención en violencia familiar. } \\
\text { - Asistencia e información al Comando de Patrullas. } \\
\text { - Módulo de capacitación (aspectos legales, } \\
\text { psicológicos y sociales) en la Escuela de O ficiales } \\
\text { de la Provincia. }\end{array}$ & . \\
\hline \multirow[t]{2}{*}{$\begin{array}{l}\text { O rganismos } \\
\text { municipales de } \\
\text { la mujer }\end{array}$} & $\begin{array}{l}\text { Programa de Violencia } \\
\text { Familiar de la Dirección } \\
\text { de la Mujer del } \\
\text { Gobierno de la Ciudad } \\
\text { de Buenos Aires }\end{array}$ & $\begin{array}{l}\text { - Centros de Atención } \\
\text { en Violencia Familiar } \\
\text { - Refugio para mujeres } \\
\text { víctimas de violencia } \\
\text { familiar. } \\
\text { - Línea telefónica pte. }\end{array}$ & $\begin{array}{l}\text { - Asistencia psicológica, grupos de ayuda mutua, } \\
\text { asesoramiento y patrocinio jurídico. } \\
\text { - Mantiene convenios con la Policía Federal para la } \\
\text { atención de casos urgentes con móvil policial. Asis- } \\
\text { tencia y vivienda para mujeres víctimas de violencia. } \\
\text { Asistencia social, atención psicológica y legal. } \\
\text { - Línea telefónica de información, asistencia y } \\
\text { contención para mujeres y niños. }\end{array}$ & $\begin{array}{l}1990 \\
1990\end{array}$ \\
\hline & $\begin{array}{l}\text { Unidades de Forta- } \\
\text { lecimiento Familiar- } \\
\text { Areas Municipales } \\
\text { Provinciales (en algunos } \\
\text { casos, coordinados } \\
\text { o dependientes de } \\
\text { la Secretaría de Acción } \\
\text { Social del Municipio) }\end{array}$ & $\begin{array}{l}\text { - Servicios de Atención } \\
\text { en Violencia Familiar }\end{array}$ & $\begin{array}{l}\text { Asistencia psicológica, grupos de ayuda mutua, } \\
\text { asesoramiento legal (en algunos casos, } \\
\text { asistencia social). }\end{array}$ & $\begin{array}{r}\text { Diversos, } \\
\text { a partir de } \\
1989\end{array}$ \\
\hline Sector Salud & $\begin{array}{l}\text { - Hospitales Generales. } \\
\text { - Red de Maltrato y } \\
\text { Violencia de la Dirección } \\
\text { de Salud Mental de la } \\
\text { Ciudad de Buenos Aires. } \\
\text { Centros de Salud/ } \\
\text { Unidades Sanitarias. }\end{array}$ & $\begin{array}{l}\text { - Equipos de atención } \\
\text { en violencia doméstica. } \\
\text { - Centros de Salud } \\
\text { dependientes de las } \\
\text { áreas programáticas } \\
\text { de los hospitales } \\
\text { generales. }\end{array}$ & $\begin{array}{l}\text { - Atención psicológica y médico asistencial } \\
\text { (principalmente) y asesoramiento } \\
\text { y orientación jurídica. } \\
\text { - Coordinación de actividades entre las } \\
\text { áreas de violencia hospitalaria y con el área } \\
\text { de violencia de la Dirección de Educación } \\
\text { de la Ciudad de Buenos Aires. } \\
\text { - Atención primaria. Se atienden los casos } \\
\text { de violencia, junto con otras problemáticas. } \\
\text { En algunos casos, se dispone de personal } \\
\text { capacitado y, en otros, se contiene y deriva. }\end{array}$ & 1993 \\
\hline Universidad & $\begin{array}{l}\text { - Universidad de } \\
\text { Buenos Aires. } \\
\text { Facultad de Psicología. } \\
\text { - Escuela de } \\
\text { Salud Pública. }\end{array}$ & $\begin{array}{l}\text { - Carrera de Posgrado } \\
\text { en Especialización } \\
\text { en Violencia Familiar. } \\
\text { - Centro de Capaci- } \\
\text { tación y Servicio para } \\
\text { atención de mujeres } \\
\text { maltratadas. }\end{array}$ & $\begin{array}{l}\text { - En sus inicios, se brindó asistencia, pero } \\
\text { actualmente sólo se cumplen actividades } \\
\text { académicas: formación de especialistas } \\
\text { en Atención en Violencia Familiar. } \\
\text { - Formación y capacitación para la Atención } \\
\text { en Violencia Familiar, y Servicio de Atención } \\
\text { para la población en general (actualmente } \\
\text { discontinuado) }\end{array}$ & 1984 \\
\hline $\begin{array}{l}\text { O rganismos } \\
\text { No guberna- } \\
\text { mentales }\end{array}$ & $\begin{array}{l}\text { Distintos organismos } \\
\text { comunitarios y de la } \\
\text { sociedad civil disponen } \\
\text { de servicios de asistencia } \\
\text { en violencia doméstica } \\
\text { en Capital Federal y } \\
\text { Gran Buenos Aires. }\end{array}$ & & $\begin{array}{l}\text { Algunos de ellos protagonizan actividades de } \\
\text { coordinación de redes, como la Red Argentina } \\
\text { contra la Violencia Doméstica y Sexual, coordinada } \\
\text { por la ONG “Lugar de Mujer”, o los Encuentros } \\
\text { Nacionales sobre Violencia Doméstica, organizados } \\
\text { y coordinados por la Fundación Alicia Moreau de } \\
\text { J usto, conjuntamente con la Asociación Argentina } \\
\text { de Prevención de la Violencia Familiar. }\end{array}$ & $\begin{array}{r}\text { Diversos, } \\
\text { a partir de } \\
1983 / 4\end{array}$ \\
\hline
\end{tabular}


La participación relativa de los «recursos humanos» en la conformación de los equipos resultó acorde con los servicios ofrecidos: 21 servicios de los 28 respondentes dijeron incluir psicólogas/os en sus equipos, 15, abogadas/ os y 18, asistentes sociales. Como dato a destacar se observa una muy baja participación de médicas/ os en los planteles asistenciales (Tabla 4). Por otro lado, debe mencionarse la mayoritaria participación femenina, así como la inclusión - en una gran parte de los equipos - de personal no profesional, sobretodo en los casos en que se trabaja con los llamados grupos de autoayuda, en los que son las mujeres que han sido víctimas de violencia conyugal las que los coordinan. El número de integrantes en los equipos fue variable, oscilaba entre 2 y 22 personas, siendo el valor modal de las instituciones encuestadas de 10.

Otro dato de interés se refiere al gran componente de voluntariado entre los/as trabajadores/as de los servicios de ONG, mientras que - por el contrario -, en la mayoría de las insti-

Tabla 3

Instituciones respondentes de la Encuesta Postal,

según servicios que prestan.

\begin{tabular}{lc}
\hline Servicios & Cap. Fed. y GBA $\left(^{*}\right)$ \\
\hline Asistencia/asesoramiento jurídico & $14(50 \%)$ \\
Asistencia/atención psicológica individual & $19(68 \%)$ \\
Patrocinio jurídico & $7(25 \%)$ \\
Hogar/refugio & $1(4 \%)$ \\
Atención/Servicio telefónico & $11(40 \%)$ \\
Grupos para hombres violentos & $1(4 \%)$ \\
Apoyo/asistencia de problemas sociales & $6(21 \%)$ \\
Grupos de autoayuda & $16(57 \%)$ \\
\hline
\end{tabular}

Tabla 4

Servicios respondentes de la Encuesta Postal, según

profesiones que integran los equipos de atención.

\begin{tabular}{lc}
\hline Profesionales & Cap. Fed. y GBA $(*)$ \\
\hline Psicólogas/os & $21(75 \%)$ \\
Abogadas/os & $15(54 \%)$ \\
Trabajadoras/es sociales & $18(65 \%)$ \\
Sociólogas/os-Psicólogas/os sociales & $13(46 \%)$ \\
Médicas/os & $3(11 \%)$ \\
Psicopedagogas/os & $2(7 \%)$ \\
Técnicas/os en Violencia Doméstica & $1(3.5 \%)$ \\
Otras y no profesionales & $7(25 \%)$ \\
\hline
\end{tabular}

(*) Los valores expresan el número de servicios en los que cada profesión integra el equipo. tuciones oficiales, el personal estaba rentado (una excepción a esta regla la constituye la Comisaría de la Mujer de Martinez, en la que el equipo de profesionales trabaja ad-honorem). Por otra parte, el $50 \%$ de los servicios dijeron contar con asesores externos - para capacitación, supervisión de casos, etc - , los que mayoritariamente provienían de espacios académicos como la carrera de Especialización en Violencia o las cátedras de Estudio de la Mujer de la Universidad de Buenos Aires (UBA).

\section{Las «redes institucionales»}

Como ya se anticipó, la intervención en este tema está en manos de efectores muy hetereogéneos, los que - por lo general - no trabajan coordinados entre sí. Aún al interior de un mismo sector como el oficial, las relaciones institucionales no son orgánicas ni articuladas.

Sin embargo, ese hecho contrasta con los canales de comunicación que se han ido construyendo principalmente como resultado del protagonismo de los organismos no gubernamentales (ONG). Entre ellos, merece destacarse la Red Argentina Contra la Violencia Doméstica y Sexual, impulsada, entre otras organizaciones, por "Lugar de Mujer", ONG que mantiene coordinación con grupos de todo el país, y que además integra la misma Red Latinoamericana. En el mismo sentido, pueden mencionarse los Encuentros Nacionales sobre este tema, organizados desde el año 1994 por la Fundación Moreau de Justo, conjuntamente con la Asociación Argentina de Prevención de la Violencia Familiar, surgidos en el marco de las actividades preparatorias para la Cumbre de Beijin 1995, y luego continuados (hasta la fecha, ya se han realizado cuatro).

En el sector oficial, la realización de eventos de esa naturaleza o de redes institucionales es menos frecuente, y, por lo general, está acotada a las jurisdicciones específicas (municipales o provinciales). Sin embargo, es habitual que los equipos de estas áreas concurran a los eventos organizados por los ONGs, y que éstes últimos - sobretodo cuando cohabitan en una misma jurisdicción - tomen parte en actividades oficiales.

En el sector salud del Gobierno de la Ciudad de Buenos Aires, existe una Red de Maltrato y Violencia en la que participan los hospitales del área. La red fue creada por iniciativa de la Dirección Nacional de Salud Mental, como resultado de la aparición de grupos dedicados al tratamiento de esta problemática en distintos hospitales. Se ocupa de violencia hacia la mujer y las/ os niñas/os; y, además de los vín- 
culos que mantiene al interior del sector, se relaciona con el Area deViolencia de Salud Escolar, dependiente de la Secretaría de Educación de la misma jurisdicción. Por su parte, la Policía, además de las actividades organizadas hacia el interior de la fuerza - sea provincial o federal -, ha organizado algunos eventos con áreas dedicadas a asuntos de mujer del sector público (sean ellas o no especialistas en el tema de violencia familiar). En 1995, la Policía Federal realizó las primeras Jornadas sobre Violencia, que contaron con la participación activa de las principales organizaciones no gubernamentales, áreas de gobierno y especialistas dedicados al tema. Pero, más allá de algunos convenios que mantiene con el sector oficial (Tabla 2), no se puede hablar de una articulación en red entre unas y otras.

Otro nexo importante - y extendido - se observa entre el sector académico y los servicios de violencia - sean gubernamentales o no gubernamentales - , a través de la capacitación, de supervisiones, etc. Como ya se anticipó, las referentes más citadas provienen de la Universidad de Buenos Aires, cátedras de Estudio de la Mujer o carrera de Especialización en Violencia.

Finalmente, cabe remarcar que, de acuerdo a la reglamentación de la nueva Ley de Violencia Familiar 24417, existirá un registro oficial de instituciones y servicios (del ámbito de la Capital Federal).

La «producción de datos y el uso de información»

Lamentablemente - al igual que se ha reconocido en otros países de la región (Ellsberg, 1996; Larrain \& Rodriguez, 1993) -, la información estadística es escasa, y la mayoría de los servicios no disponen de datos sistemáticos y confiables como para monitorear sus propias actividades.

La mitad de los servicios de los ONG dijeron disponer de instrumentos de registro, pero sólo uno declaró producir información estadística. Mientras que, entre los dependientes de áreas gubernamentales, todos menos uno dijeron utilizar instrumentos de registro y más de la mitad, producir estadísticas. Sin embargo, cuando se consultó sobre antecedentes en investigación fueron - en términos relativos más los servicios de los ONGs que los dependientes de organismos oficiales los que tenían algún desarrollo en la materia.

El área de la mujer del gobierno de la ciudad de Buenos Aires cuenta - prácticamente desde el momento de su fundación - con un departamento de Programas y Proyectos que, entre otras cosas, tiene a su cargo la elaboración de las planillas de registro, la sistematización, procesamiento y análisis de la información de los servicios dependientes de esa Dirección de la Mujer. El departamento dispone de instrumentos de registro, con sus repectivos instructivos, para cada uno de los distintos servicios que presta (asistencia psicológica, llamadas telefónicas, grupos, refugios, etc.), los que fueron elaborados con el apoyo de la Dirección de Estadística de esa jurisdicción. En la actualidad - por limitaciones presupuestarias y de infraestructura -, sólo se procesa una porción de la información potencialmente disponible. Pese a ello, esta institución constituye una de las fuentes más ricas y confiables de toda el área investigada.

Entre los organismos no gubernamentales (ONG) del total de instituciones relevadas, sólo uno disponía de información confiable y metodológicamente cuidada, aunque elaborada por única vez (no es producción permanente). Se trata de la Fundación Alicia Moreau de Justo. El estudio recopila información de tres años - 1989, 1990 y 1991 -, sobre una muestra de 500 casos, seleccionada aleatoriamente del total de consultas registradas (admisiones).

Conjuntamente con la producción y el uso de información, se evaluaron los instrumentos actualmente en uso. Se encontró una heterogeneidad muy grande tanto en lo que se refiere a aspectos conceptuales - variables e indicadores incluídos en los mismos -, como a los aspectos formales: desde fichas con un nivel mínimo de estructuración (más próximas a una historia clínica que a una planilla de registro) hasta instrumentos que incluían gran cantidad de ítems o indicadores, pero prácticamente ninguna precodificación de las categorías correspondientes.

Lo recurrente fue la inclusión de indicadores de evaluación socioeconómica, como condición de actividad, ocupación, educación, residencia, ingresos, características de la vivienda, etc.; de caracterización del grupo familiar: conformación del grupo, número de hijos, antecedentes de violencia en la familia de origen - de la víctima y del agresor -, vínculo que la persona consultante mantiene con el agresor, antiguedad de esa relación, etc; indicadores para la caracterización delas situaciones deviolencia: motivo de consulta (para discriminar el tipo de violencia), evaluación del riesgo (intensidad, frecuencia y tipo de agresiones), consultas previas, intervención de médico forense (que permite discriminar la exposición policial - que no implica la intervención de la justicia, 
de la denuncia, que sí tiene derivación a juzgado); en varios de los instrumentos, se incluía la pregunta por "situaciones que desencadenan violencia", senálandose especialmente el embarazo, el nacimiento de hijos, Ia celotipia, las acusaciones de infidelidad, etc. Algunos instrumentos incluían ítems referidos a la accesibilidad al servicio (p.e., preguntando por qué medio lo conoció), y muy pocos incluían referencias a la socialización de género y reparto de responsabilidades (como uso del dinero, control de gastos, atención de niños, distribución de tareas domésticas, etc.).

En relación a este punto, merece señalarse que, en el marco del Programa en Violencia Familiar iniciado por el Consejo Nacional de la Mujer y UNICEF, se está elaborando un instrumento de registro que incorpora la opinión y las recomendaciones de instituciones gubernamentales, no gubernamentales y académicas. Se realizaron para ello talleres de trabajo, y la propuesta resultante será puesta a prueba durante el año 1997.

También el programa desarrollado por la Oficina Sanitaria Panamericana, denominado "The Critical Process", ha reconocido la necesidad de mejorar los registros y la producción de información, y ha incluído un capítulo para el tratamiento del tema. Argentina forma parte de los países beneficiarios del mismo. La primera experiencia ha sido iniciada en la provincia de Mendoza, bajo la coordinación del Ministerio de Salud de la Nación.

Por otro lado, en la reglamentación de la Ley 24417 de Violencia Familiar, se ha estipulado un formulario para el registro de denuncias, por agresor y por víctima. El Consejo Nacional del Menor y de la Familia tendrá a su cargo el procesamiento y la difusión de esa información.

\section{Caracterización de la población asistida}

Además de la evaluación que se puede hacer a través de la información estadística que, como se anticipó, es muy escasa, se solicitó a los servicios encuestados que describieran a las mujeres por ellos asistidas según dos dimensiones de análisis: a) por referencia a variables de índole socio-económica, y b) según una caracterización psico-social. En ambos casos se pedía una valorización cualitativa, que podía acompañarse o no de información cuantitativa.

La mayor heterogenidad se registró en la caracterización socioeconómica, ya que las respuestas iban desde quienes afirmaban que «concurrían mujeres de todo el espectro social» a las que asistían a «mujeres de escasos recursos, con bajo nivel de instrucción»e inclusive con «riesgo psicosocial». Ello puede deberse a que el perfil de la población asistida varía de acuerdo a la ubicación geográfica de la institución, mientras que los determinantes de tipo psicológico - como se reconoce en la literatura existente (Corsi, 1994; Ferreira, 1992) parecen no variar significativamente por clase social.

En cuanto a las determinantes de tipo psicológico, la constante fue el reconocimiento de que se trata de mujeres con baja autoestima, que llegan atemorizadas y paralizadas por las situaciones que viven, dependientes, abandonadas, con tendencia a autoculpabilizarse. En algunos casos, se enfatizaba también que ese perfil psicológico es afín a los estereotipos de género imperantes. Varias encuestadas remarcaron que la mujer víctima de violencia no debería ser encasillada psicopatológicamente, sino que, por el contrario, debía ponerse el acento en la exposición prolongada a situaciones de maltrato, por considerar a éste el factor conducente al desequilibrio emocional.

Por otro lado, una calificada especialista entrevistada hizo referencia a la construcción de "tipos maternales" que suelen ser antecedentes o coadyuvantes familiares en los casos de violencia: el de la "Madre Siniestra”, que corresponde a un tipo de mujer autoritaria, cuando se apela al castigo como forma de autoridad y poder en la familia, y que reproduce el modelo de la violencia con sus propios/ as hijos/ as; o la "Madre Sometida" que, además de mostrarse sumisa en la relación con su cónyuge, suele ser agredida por sus propios hijos varones cuando éstos llegan a la edad adolescente.

Aunque la información estadística sea escasa y fragmentaria como para contrastar las afirmaciones precedentes, pueden presentarse algunos datos a modo ilustrativo. En la Figura 1, se observa la evolución de la cantidad de llamadas recibidas por el Servicio de Violencia Familiar de la Dirección de la Mujer de la Ciudad de Buenos Aires, durante los años 1990 a 1996. El acusado aumento que se registra a partir del año 1995 coincide con la implementación de campañas de difusión iniciadas por esa institución. Ello da cuenta de la importancia de la difusión en este tema, particularmente en lo que se refiere a información sobre servicios. La Figura 2 permite confirmar la cronicidad que suele presentar este problema: el $60 \%$ de los casos que llamaron a lo largo del período llevaban más de 5 años de exposición a situaciones de violencia, y el 40\%, más de 11 . El informe "La Violencia Conyugal en Cifras", elaborado por la "Fundación Moreau de Justo", 
ofrece información sobre una muestra de 500 mujeres concurrentes a ese servicio. En él se constata la alta concentración de mujeres en edades medias (30 a 40 años); una gran concentración de mujeres con hijos - lo que se verifica si se la compara con la distribución que presenta el Censo de Población 1991 (Figura 3) y con un dato que coincide con lo señalado en otras investigaciones sobre el tema (Ellsberg, 1996): el hecho de tener un cónyuge o compañero con antecedentes de violencia en su familia de origen resulta influir mucho más en el riesgo de ser una mujer maltratada que el haber tenido una historia de maltratos. En este estudio casi el $83 \%$ de los agresores habían sido testigos o víctimas de violencia en su familia de origen; frente a sólo el $22 \%$ de las mujeres consultantes. El gran porcentaje de amas de casa (40\%) parece abonar la hipótesis de que esa condición es uno de los factores que aumentan el riesgo al maltrato. Sin embargo, esas cifras se relativizan si se las compara con el número de mujeres económicamente inactivas de la población general: 33\% en Capital Federal y $49 \%$ en Gran Buenos Aires (para el rango de edad comprendido en el estudio de referencia - 20 a 59 años -, y según el Censo de Población de 1991).

\section{Concepciones teóricas en torno a la} problemática de la violencia familiar

Un primer aspecto a destacar es la existencia de distintos enfoques teóricos por parte de los equipos dedicados a este tema. A partir de la información recopilada, pudieron identificarse dos grandes tipos generales en las concepciones con que se definía la violencia familiar, y en particular la dirigida contra la mujer, los que, a su vez, presentaban algunas variaciones entre sí. Por un lado - aunque minoritarios -, estaban los enfoques que cabría denominar «universalistas», que reconocen a la violencia como un fenómeno social estructural. En ellos no se señalan diferencias cualitativas en lo que respecta a la violencia dirigida contra la mujer. Según esas definiciones, la etiología de la violencia hay que buscarla en determinantes sociales - como la marginación, el desempleo, la falta de educación, el autoritarismo que impera en la sociedad - que como tales pueden afectar, al menos potencialmente, a unos y a otras, indistintamente. El otro tipo se caracteriza por la explícita inclusión de la concepción de género. También en éste la etiología del problema se reconoce como social, pero se describe lo específico de la violencia hacia la mujer, poniendo el énfasis en las desigualdades de poder
Figura 1

Evolución de la cantidad de llamadas del Servicio de Violencia Familiar, del Gobierno de la Ciudad de Buenos Aires. 1990 a 1996*.

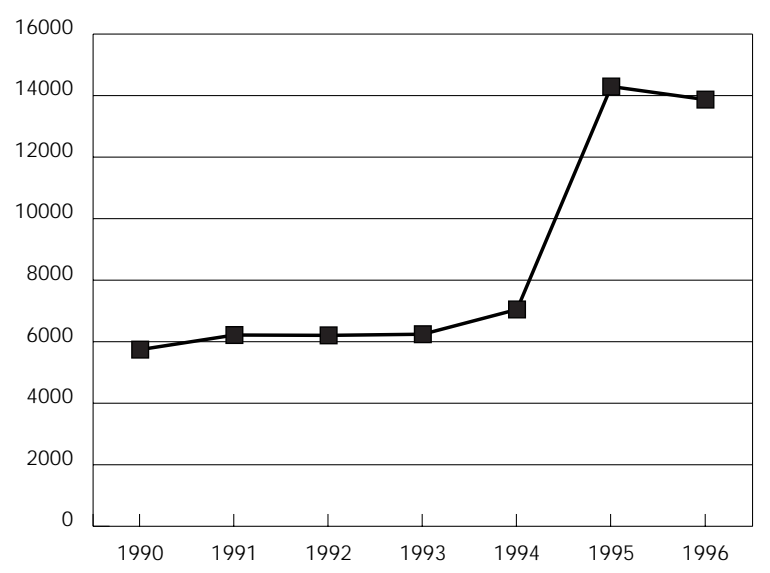

* En este servicio se recepcionan llamadas de contención, información y emergencia. Fuente: Elaboración propia en base a Informe sobre Servicios de Atención en Violencia Doméstica de la Dirección de la Mujer, del Gobierno de la Ciudad de Buenos Aires.

Figura 2

Distribución porcentual de las llamadas según tiempo de exposición al maltrato de mujer consultante*.

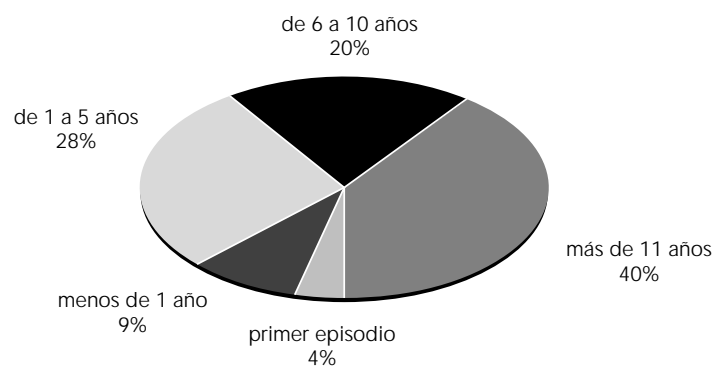

* Total de llamadas acumuladas 1990 - 1996: 59622.

Fuente: Idem Figura 1.

existentes entre varones y mujeres. Se mencionan además los determinantes culturales que sostienen esa asimetría (educación patriarcal, subordinación histórica de la mujer, mitos y estereotipos, etc.), señalando que ellos atraviesan todos los niveles sociales e invisibilizan los fenómenos de violencia de género.

De cada una de estas concepciones, se derivaban distintas prácticas de intervención. En torno al último tipo, era frecuente encontrar el trabajo en grupos de autoayuda (coordinados por mujeres que fueron víctimas de violencia) 
Figura 3

Distribución porcentual de mujeres de 20 a 59 años, según el número de hijos. Censo Nacional de Población 1991 (Capital Federal y Gran Buenos Aires) y población asistida en el Servicio de Violencia Familiar de la Fundación Moreau de J usto, años 1989, 1990 y 1991.

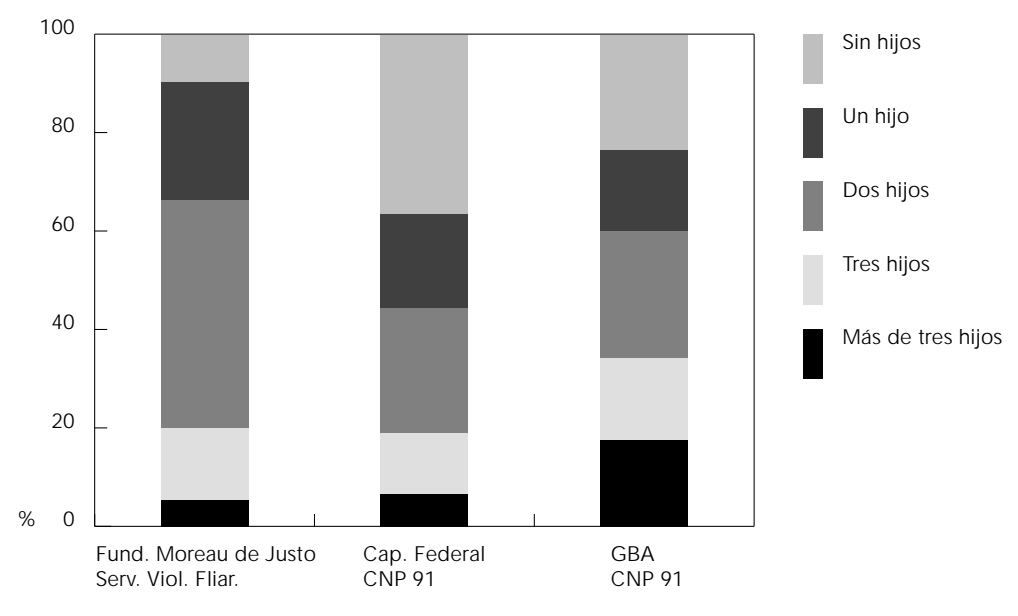

Fuente: Elaboración propia en base al Informe "La Violencia Conyugal en Cifras", de la Fundación Alicia Moreau de J usto, y al Censo Nacional de Población Argentina 1991-INDEC. o de ayuda mutua (coordinados por profesionales); a veces combinado con la entrevista o tratamiento psicológico individual. También la inclusión de grupos con "hombres violentos" se verificó en un organismo que adhería al enfoque de género, por considerar que, precisamente, las cuestiones de género atañen a varones y mujeres, y ambos deben ser tratados; además, el tratamiento con el «agresor» apuntaba a evitar que él vuelva a reeditar situaciones de violencia con otra o con la misma mujer. Por otro lado, entre los enfoques con menos énfasis en los componentes de género, se priorizaban los estudios psicodiagnósticos y/o se tendía a trabajar con el grupo familiar (incluyendo al sujeto golpeador o agresor).

Es importante señalar que estas tipologías no se presentaron asociadas con la dependencia institucional de los servicios. Tanto entre los ONGs como entre los organismos gubernamentales, se puedieron identificar posiciones cercanas a unos como a otros.

Además de los tipos señalados, las definiciones de la violencia incluyeron otras dimensiones conceptuales. Algunas consideraron a la violencia como «delito»y como violación de los derechos humanos. En un sólo caso se la consideró como una «enfermedad que se debe tratar», asociada al consumo de alcohol.

Una vía complementaria para la caracterización del enfoque conceptual se recogió a tra- vés del ítem de "objetivos fundacionales del servicio". Ellos importan particularmente para evaluar la relación existente entre Organismos No Gubernamentales y Estado. En la literatura, se ha señal ado (Andrew, 1995) la existencia de distintas posiciones en lo que hace a la competencia de las áreas gubernamentales para abordar la problemática de la mujer y el género. Por una parte estarían quienes consideran que la atención de estos temas son funciones propias de los ONGs (posición que entraña además cierta desconfianza ideológica hacia los aparatos de Estado); mientras que, por otro, estarían quienes consideran que la vía formal - de las áreas de gobierno - puede complementarse con la vía informal de los organismos no gubernamentales.

En el caso argentino - al menos evaluado a la luz de este relevamiento -, esa disyuntiva es menos clara, y lo que se observa es una posición de «reclamo» hacia el sector oficial. Un importante porcentaje de organismos no gubernamentales (ONG) sostenían que habían creado el "Servicio en Violencia", para dar respuesta a una demanda que surgía de las mujeres y para la cual el Estado no disponía de recursos ni de políticas. También las áreas gubernamentales dedicadas a los asuntos de la mujer reconocen que la creación del servicio nace para dar respuesta a una demanda creciente. Pero - como lo muestran lo datos históricos lo cierto es que fueron las organizaciones no gubernamentales, impulsadas por el movimiento de mujeres, quienes tuvieron la delantera en estos temas.

\section{Obstáculos y necesidades de los servicios}

Una gran parte de las instituciones encuestadas - principalmente los ONGs - reconocieron como tema crítico la falta de fondos económicos. Haciendo referencia a los problemas existentes para conseguir apoyos y subsidios, y señalando además que, aún entre los organismos no gubernamentales (ONG), hay algunos pocos que «monopolizan» la captación de ese tipo de beneficios.

Otra necesidad reconocida por la casi totalidad de los servicios estuvo relacionada con la falta de "Refugios" y "Hogares" para albergar a las mujeres víctimas de violencia; transfórmandose este déficit - según muchas de ellos en uno de los obstáculos para «romper el ciclo de violencia».

Se refirieron también a un problema que llamaron "segunda victimización" (y que, desde una perspectiva menos "victimizadora", podría denominarse "violencia institucional"). 
Con él se aludía a las múltiples derivaciones y a la desinformación de que son objeto, principalmente por parte de servicios e instituciones del ámbito policial, judicial e inclusive sanitario, las mujeres víctimas de violencia familiar que consultan a cualquiera de dichos servicios.

En lo que respecta a la fuerza policial, y pese a los avances institucional es que se han ido consiguiendo (Tabla 2), los déficits aún son también muy grandes. Lo habitual es que, en la mayoría de los casos, cuando las mujeres llegan a las comisarías generales, se les tome una "exposición policial", de la que, a diferencia de la denuncia, no se sigue ninguna acción judicial; y ello sin dar información ni elección a las implicadas.

Una mención reiterada fue la poca institucionalización de las políticas y programas oficiales, sujetos a las voluntades de funcionarios/ as de turno, lo que conduce a discontinuar los programas en cada recambio de gestión. También se mencionó la falta de «redes» que faciliten el acceso a la información, bibliografía y referencias sobre recursos disponibles a nivel nacional e internacional, mientras que, en otros casos, se señaló la falta de campañas masivas tendientes a difundir el tema y concientizar las personas.

Además de los obstáculos que atañen al funcionamiento institucional, se señalaron los que son propios de la problemática, y que aparecen como límites en la intervención: los altos índices de deserción y la recurrencia («ciclo de la violencia»), la falta de trabajo y las limitaciones económicas que impiden a las mujeres independizarse.

\section{Conclusiones}

Como se desprende de lo expuesto, gran parte de los déficits y necesidades señaladas son efecto de la falta casi absoluta de políticas y programas capaces de integrar y coordinar las acciones en marcha.

La planificación de los mismos requerirá de una visión innovadora, capaz de incorporar el desarrollo y la tradición institucional existentes. Ello supone la articulación entre áreas que fueron históricamente «autosuficientes», como las de salud o justicia; y la inclusión de sectores que han resultado muchas veces conflictivos para las burocracias estatales, como las organizaciones de la comunidad.

En lo que respecta al campo sanitario, exige también que se asuma a la atención en salud inclusive la biomédica -, más allá del trata- miento de lesiones o síntomas, para dirigir la mirada a las tramas y vínculos sociales que unen la persona con su entorno (OPS, 1993). El sector salud debiera transformarse en uno de los polos más sensibles para la detección y orientación de casos de violencia familiar (distintos estudios señalan un al to porcentaje de maltrato, muchas veces encubierto, entre las mujeres que se presentan en los servicios de emergencia o consultorios externos de distintas especialidades - Heise, 1994).

Pese a todos los déficits existentes, y como se desprende de la información presentada, el tema se ha instalado y ha hecho posible el encuentro, sino formal al menos informal, entre representantes de todos esos sectores. En tal sentido, y aunque resta mucho por hacer, el caso de la violencia hacia la mujer - y el de la violencia familiar, en general - podría ser considerado como un ejemplo de las nuevas articulaciones institucionales que resultan del protagonismo de la comunidad (y de los actores que la representan), conjuntamente con las organizaciones de la sociedad civil y con el Estado.

El análisis de esas «nuevas articulaciones» requiere un desarrollo teórico que excede los alcances de este escrito. Sin embargo, pueden sugerirse algunos conceptos para la comprensión de las distintas formas institucionales, y del papel de la mujer en cada una de ellas.

La descripción de la sociedad según un orden comunal-familiar, uno societal y otro político ha sido propugnada por distintas corrientes filosófico-políticas (Hegel, 1988; Marx, 1973; Durkheim, 1982; Giddens, 1995). Ella supone que “...el conjunto de las relaciones sociales [podría ser definido según] tres grandes módulos: 1) las relaciones comunales, propias del contexto familiar, y de sus relaciones territoriales inmediatas, en la forma de vecindario; 2) las relaciones societales, propias del contexto contractual, interindividual, y que se constituye por el movimiento del mercado y de las asociaciones que él hace posible; y 3) las relaciones políticas, propias del contexto estatal o de la centricidad contractual" (Samaja, 1993).

Esas tres formas, aunque reconozcan una genésis de desarrollo, se encuentran presentes en las sociedades contemporáneas. Constituyen el hábitat natural de la vida humana, ya que en ellas se despliega el universo de vínculos que unen a la persona con su entorno: como sujetos del ámbito comunal (familiar) en el que se consuma su «socialización primaria»-, como individuos habilitados para la contractualidad societal y como ciudadanos que toman parte en las cuestiones ético-políticas que forjan su destino histórico. 
En lo que respecta a la problemática de género, este modelo se torna particularmente útil para describir los distintos planos en los que se expresan las relaciones de poder intergenéricas; si se tiene presente que las vinculaciones entre ellos no son lineales. Por ejemplo, la salida de la mujer al mundo extradoméstico, si bien significó grandes cambios en las relaciones entre los géneros, no se tradujo en una necesaria liberación de los sometimientos a que estaba sujeta en la vida del hogar, y además la enfrentó con nuevas discriminaciones (las que en el mundo societal están siempre combinadas con los determinantes propios de la clase social de pertenencia).

Por otro lado, el mismo modelo de los planos de sociabilidad (comunal-societal-político) se puede aplicar para describir los procesos con los que se «responde» a aquello que aparece como «situación-problema», y las resistencias e intereses diversos que se ponen en juego en ellas. Esta es la dimensión que corresponde al análisis institucional, y que, en particular, se puede utilizar para el caso que nos ocupa.

En lo que a la violencia de género respecta, fue el movimiento de mujeres el que puso en cuestión un fenómeno «naturalizado»por siglos (no sólo de hecho sino también de derecho, como lo expresan códigos y normativas de casi todas las épocas (Optiz, 1992; ArnaudDuc, 1993). Ello supuso la intervención en varios planos al mismo tiempo: a nivel de los sistemas de valores imperantes, en las instituciones de la sociedad civil y en los aparatos y órdenes jurídicos de Estado (locales, nacionales e internacionales). Las organizaciones de mujeres que lideraron ese proceso no sólo salieron a buscar «asistencia». Forjaron sus propias estrategias de intervención (haciendo necesaria la revisión de enfoques disciplinarios y teóricos, como los del campo de la salud mental) y se tranformaron en actores políticos a los que el Estado debió escuchar, e inclusive consultar e incorporar, para el diseño de acciones y políticas en la materia (aún cuando ese acercamiento haya sido fluctuante y titubeante, como lo indica el análisis de las experiencias concretas).

Teniendo presente que esas reivindicaciones y conquistas se dieron en el marco de la consolidación del modelo neoliberal (con el consecuente abandono de las políticas sociales y sanitarias por parte de los estados) y durante la Ilamada «crisis de representación», su importancia es aún más significativa. Ello sin desconocer que, por otro lado, este tema apareció muchas veces como políticamente más «potable»- en particular para los gobiernos locales -; por su gran potencial de movilización (sobretodo en momentos de conmoción de la opinión pública, cuando se conocía alguna muerte trágica producto de violencia familiar) y por ser un tema de atracción multiclasista.

Aunque no es posible hablar de una verdadera articulación orgánica entre el Estado (y sus estructuras en distintos niveles) y las organizaciones de mujeres y la comunidad, debe reconocerse - sin embargo - que la lucha contra la violencia de género fue instalada como tema de agenda pública por la presión ejercida desde esas organizaciones. Se diferencía, por lo tanto, de otras experiencias de supuesta participación comunitaria, que fueron inducidase impulsadas por los propios poderes nacionales e internacionales como estrategias destinadas a desplazar las responsabilidades del Estado (Testa \& Bloch, 1988). Y también de las que integraron a las mujeres para utilizar su mano de obra impaga, incrementando así la eficiencia de programas, y reduciendo al mínimo el gasto social (De los Rios, 1993).

Por todo ello, el protagonismo del movimiento de mujeres y de las organizaciones de la comunidad en la institucionalización de esta problemática y el impacto socio-cultural que ellas acarrearon en distintos ámbitos de la sociedad constituyen las conclusiones más importantes a remarcar. 


\section{Agradecimientos}

La investigación contó con la colaboración permanente de la Lic. Graciela Colombo.

\section{Referencias}

ANDREW, C., 1995. El gobierno municipal y el movimiento de mujeres. Travesías, 3:53-73.

ARNAUD-DUC, N., 1993. Las contradicciones del derecho. In: Historia delas Mujeres. (G. Duby \& M. Perrot, comp.), pp. 91-127 (Tomo 7). Madrid: Ed.Taurus.

CORSI, J., 1994. Violencia Familiar. Una Mirada Interdisciplinaria sobre un Grave Problema Social. Buenos Aires: Ed.Paidós.

DE LOS RIOS, R., 1993. Género, Salud y Desarrollo: un enfoque en construcción. In: Mujer, Salud y Desarrollo en las Américas (Organización Panamericana de la Salud, org.), pp. 3-18. Washington: OPS.

DURKHEIM, E., 1982. La División del Trabajo Social. Madrid: Ed. Akal.

FERREIRA, G., 1992. Hombres Violentos, Mujeres Maltratadas: Aportes a la Investigación y Tratamiento de un Problema Social. Buenos Aires: Ed.Sudamericana.

GIDDENS, A., 1995. La Constitución de la Sociedad. Bases para la Teoría de la Estructuración. Buenos Aires: Ed. Amorrortu.

ELLSBERG, M., 1996. Confites en el Infierno. Prevalencia y Características de la Violencia Conyugal Hacia Las Mujeres en Nicaragua. Managua: Ed. UNAN-León.

HEGEL, G. W. F., 1988. Principios de la Filosofía del Derecho. Barcelona: Ed. Edhasa.

HEISE, L., 1994. Violencia Contra la Mujer: La Carga Oculta de Salud. Programa Salud, Mujer y Desarrollo. Washington: OPS.
LARRAIN, S. \& RODRIGUEZ, T., 1993. Los orígenes y el control de la violencia doméstica en contra de la mujer. In: Mujer, Salud y Desarrollo en las Américas (Organización Panamericana de la Salud, org.), pp. 202-209. Washington: OPS.

MARX, C., 1973. Elementos Fundamentales para Ia Crítica dela Economía Política (Borradores 18571858). Buenos Aires: Ed. Siglo XXI.

NIEVES RICO, M., 1992. Violencia Doméstica contra la Mujer en América Latina y el Caribe: Propuesta para la Discusión. Santiago de Chile: División de Desarrollo Social. Unidad Mujer y Desarrollo, CEPAL.

OPS (Organización Panamericana de la Salud), 1993. La Violencia Contra las Mujeres y las Niñas: Análisis y Propuestas desde la Perspectiva de la Salud Pública. MSD13/6.13a Reunión. Washington: OPS.

OPTIZ, C., 1992. Vida cotidiana de las mujeres en la Baja Edad Media (1250-1500). In: Historia de las Mujeres. (G. Duby y M. Perrot, comp.), pp. 321 393 (Tomo 3). Madrid: Ed. Taurus.

SAM AJA, J., 1993. La reproducción social y la relación entre la salud y las condiciones de vida. Monografía OPS, inédita.

TESTA, M. \& BLOCH, C., 1988. Estado y Salud. Publicación № 5. Buenos Aires: Representación de Argentina - Organización Panamericana de la Salud. 